\title{
Correction to: Mussel biology: from the byssus to ecology and physiology, including microplastic ingestion and deep-sea adaptations
}

\author{
Koji Inoue $^{1}$ [ $\cdot$ Yuri Onitsuka ${ }^{2,1} \cdot$ Tomoko Koito $^{3}$
}

Published online: 28 October 2021

(c) Japanese Society of Fisheries Science 2021

\section{Correction to: Fisheries Science \\ https://doi.org/10.1007/s12562-021-01550-5}

In the Original Publication, an article note is missed and it should be "Published with support by the Japan Society for the Promotion of Science (JSPS) KAKENHI Grant no. JP19HP2002".

The Article note is available in this correction.

Publisher's Note Springer Nature remains neutral with regard to jurisdictional claims in published maps and institutional affiliations.

The original article can be found online at https://doi.org/10.1007/ s12562-021-01550-5.

Koji Inoue

inouek@aori.u-tokyo.ac.jp

Yuri Onitsuka

7127933519@edu.k.u-tokyo.ac.jp

Tomoko Koito

koito.tomoko@nihon-u.ac.jp

1 Atmosphere and Ocean Research Institute, The University of Tokyo, Kashiwa, Japan

2 Graduate School of Frontier Sciences, The University of Tokyo, Kashiwa, Japan

3 Department of Marine Science and Resources, College of Bioresource Sciences, Nihon University, Fujisawa, Japan 